PROCEEDINGS OF THE

AMERICAN MATHEMATICAL SOCIETY

Volume 138, Number 4, April 2010, Pages 1223-1230

S 0002-9939(09)10171-5

Article electronically published on December 4, 2009

\title{
ON THE VALUES OF A CLASS OF DIRICHLET SERIES AT RATIONAL ARGUMENTS
}

\author{
K. CHAKRABORTY, S. KANEMITSU, AND H.-L. LI
}

(Communicated by Ken Ono)

Dedicated to Professor Eiichi Bannai on his sixtieth birthday, with great respect and friendship

\begin{abstract}
In this paper we shall prove that the combination of the general distribution property and the functional equation for the Lipschitz-Lerch transcendent capture the whole spectrum of deeper results on the relations between the values at rational arguments of functions of a class of zeta-functions. By Theorem 1 and its corollaries, we can cover all the previous results in a rather simple and lucid way. By considering the limiting cases, we can also deduce new striking identities for Milnor's gamma functions, among which is the Gauss second formula for the digamma function.
\end{abstract}

\section{Introduction And the finite Value CASE}

Srivastava [S2] (cf. also Srivastava and Choi [SC], pp. 336-344) gives two simpler proofs of interesting results of Cvijović and Klinowski [CK1] on the finite expression for the values of a Bernoulli polynomial at rational arguments in terms of the values of the Hurwitz zeta-function at integer arguments with rational values for the perturbation.

The first proof uses the Fourier series for the periodic Bernoulli polynomial and the formula for the decomposition into residue classes. The second proof uses the functional equation for the partial zeta-function

$$
\zeta\left(1-s, \frac{p}{q}\right)=\frac{2 \Gamma(s)}{(2 \pi q)^{s}} \sum_{a=1}^{q} \cos \left(\frac{\pi s}{2}-\frac{2 \pi a p}{q}\right) \zeta\left(s, \frac{a}{q}\right)
$$

([SC, (8), p. 90], Ap ) to be restated as (1.11) below, where

$$
\zeta(s, a)=\sum_{n=0}^{\infty} \frac{1}{(n+a)^{s}}, \sigma>1,
$$

is the Hurwitz zeta-function.

We introduce the Lipschitz-Lerch transcendent

$$
L(s, z, a)=\sum_{n=0}^{\infty} \frac{e^{2 \pi i n z}}{(n+a)^{s}}
$$

Received by the editors December 28, 2008, and, in revised form, August 18, 2009

2010 Mathematics Subject Classification. Primary 11M35; Secondary 33B15.

Key words and phrases. Lipschitz-Lerch transcendent, Hurwitz zeta function, polylogarithm function, Gauss' second formula, Milnor's gamma function.

(c)2009 American Mathematical Society Reverts to public domain 28 years from publication 
absolutely convergent for $\sigma>1$ if $z=x \in \mathbb{R}$ or $s \in \mathbb{C}$ with $\operatorname{Im} z>0$, which generalizes not only the Hurwitz zeta-function

$$
\zeta(s, a)=L(s, 1, a)
$$

but also the polylogarithm function (or the Lerch zeta-function)

$$
l_{s}(x)=L(s, x, 1)=\sum_{n=1}^{\infty} \frac{e^{2 \pi i n x}}{n^{s}}, \sigma>1 .
$$

The most general distribution property of the Hurwitz-Lerch transcendent (which follows from the principle of decomposition into residue classes used in Srivastava's first method),

$$
L(z, s, a)=q^{-s} \sum_{j=0}^{q-1} L\left(q z, s, \frac{a+j}{q}\right) e^{2 \pi i j z},
$$

([SC, (15), p. 339]) coupled with the functional equation for the Lipschitz-Lerch transcendent ([SC, (29), p. 125]),

$$
L(x, 1-s, a)=\frac{\Gamma(s)}{(2 \pi)^{s}}\left\{e^{\pi i\left(\frac{1}{2} s-2 a x\right)} L(-a, s, x)+e^{-\pi i\left(\frac{1}{2} s-2 a(1-x)\right)} L(a, s, 1-x)\right\},
$$

captures the whole scene and gives an immediate proof of new limiting relations as well as all these types of linear combination expressions for a class of zeta-functions and as a special case for polynomials.

Theorem 1. For $1<q \in \mathbb{Z}$ with $0<x<1$ and $\operatorname{Re} a>0$, one has the following identity:

$$
\begin{aligned}
& q^{-s} \sum_{j=0}^{q-1} L\left(q x, s, \frac{a+j}{q}\right) e^{2 \pi i j x}=L(x, s, a)=\frac{\Gamma(1-s)}{(2 \pi)^{1-s}} \\
& \times\left\{e^{\pi i\left(\frac{1}{2}(1-s)-2 a x\right)} L(-a, 1-s, x)+e^{-\pi i\left(\frac{1}{2}(1-s)-2 a(1-x)\right)} L(a, 1-s, 1-x)\right\}
\end{aligned}
$$

except at the singularities of $L$, in which case, the identity is to mean the limit.

Specializing the parameter $a$ suitably, Theorem 1 reads:

Corollary 1. For $1<q \in \mathbb{Z}$ and $q>p \in \mathbb{N}$, we have the identity

$$
\begin{aligned}
q^{-s} & \sum_{a=1}^{q} e^{2 \pi i \frac{a}{q} p} \zeta\left(s, \frac{a}{q}\right)\left(=l_{s}\left(\frac{p}{q}\right)\right) \\
& =i \frac{\Gamma(1-s)}{(2 \pi)^{1-s}}\left\{e^{-\frac{\pi i s}{2}} \zeta\left(1-s, \frac{p}{q}\right)-e^{\frac{\pi i s}{2}} \zeta\left(1-s, 1-\frac{p}{q}\right)\right\},
\end{aligned}
$$

where, for $s=0$ or $s=1$, the identity is to mean the limit as $s \rightarrow 0$ or $s \rightarrow 1$.

We shall show that (1.9) and its counterpart for the polylogarithm function already entail not only all the previous results but also new non-trivial corollaries. As an example, we note that the trigonometric series $(\nu>1)$

$$
C_{\nu}(\pi \alpha)=\sum_{k=0}^{\infty} \frac{\cos (2 k+1) \pi \alpha}{(2 k+1)^{\nu}}, S_{\nu}(\pi \alpha)=\sum_{k=0}^{\infty} \frac{\sin (2 k+1) \pi \alpha}{(2 k+1)^{\nu}},
$$


introduced by Srivastava and Choi ([SC, (1), p. 341]), may be thought of as the real and imaginary parts of the odd part of

$$
l_{s}\left(\frac{\alpha}{2}\right)-2^{-s} l_{s}(\alpha) .
$$

Hence by the general distribution property (1.6) one has

$$
\begin{aligned}
C_{s}\left(\pi \frac{p}{q}\right)+i S_{s}\left(\pi \frac{p}{q}\right) & =l_{s}\left(\frac{p}{2 q}\right)-2^{-s} l_{s}\left(\frac{p}{q}\right) \\
& =(2 q)^{-s} \sum_{j=1}^{q} \zeta\left(s, \frac{2 j-1}{2 q}\right) e^{2 \pi i \frac{2 j-1}{2 q} p} .
\end{aligned}
$$

This proves $[\mathrm{SC},(8),(9)$, p. 342], which are equivalent to Cvijović-Klinowski results CK2] (cf. [S1]).

Also note that in view of

$$
C_{2 \nu-1}(\pi \alpha)=\frac{(2 \pi)^{2 \nu-1}(-1)^{\nu-1}}{(2 \nu-1) !}\left(C l_{2 \nu-1}\left(\frac{\alpha}{2}\right)-2^{-(2 \nu-1)} C l_{2 \nu-1}(\alpha)\right)
$$

and

$$
S_{2 \nu}(\pi \alpha)=\frac{(2 \pi)^{2 \nu}(-1)^{\nu}}{(2 \nu) !}\left(C l_{2 \nu}\left(\frac{\alpha}{2}\right)-2^{-2 \nu} C l_{2 \nu-1}(\alpha)\right)
$$

the statement by Srivastava-Choi about the slow convergence of the series is justified. Here $C l_{\nu}(\alpha)$ denotes the Clausen function (log-sine integrals; cf. [L], $[\mathbf{Y}$, [K]) defined as

$$
C l_{\nu}(\alpha)= \begin{cases}\sum_{k=1}^{\infty} \frac{\sin k \alpha}{k^{\nu}}, & \text { if } 2 \mid n, \\ \sum_{k=1}^{\infty} \frac{\cos k \alpha}{k^{\nu}}, & \text { if } 2 \not n .\end{cases}
$$

Corollary 2 ( $(\mathrm{Ap}$, Theorem 128, p. 261], SC, (8), p. 90]). For all $s \neq 0,1$, we have

$$
\zeta\left(1-s, \frac{p}{q}\right)=\frac{2 \Gamma(s)}{(2 \pi q)^{s}} \sum_{a=1}^{q} \cos \left(\frac{\pi s}{2}-\frac{2 \pi a p}{q}\right) \zeta\left(s, \frac{a}{q}\right),
$$

and for $n>1,0 \leq p \leq q$,

$$
B_{n}\left(\frac{p}{q}\right)=-\frac{2 n !}{(2 \pi q)^{n}} \sum_{j=1}^{q} \cos \left(\frac{2 \pi j p}{q}-\frac{n \pi}{2}\right) \zeta\left(n, \frac{j}{q}\right) .
$$

Proof. To prove (1.11) we sum two equalities: one is $e^{-\frac{\pi i s}{2}}$ times (1.9), and the other is $e^{\frac{\pi i s}{2}}$ times (1.9) with $p$ replaced by $q-p$. Then we get

$$
\begin{gathered}
q^{-s} \sum_{a=1}^{q}\left(e^{\left(-\frac{\pi s}{2}+\frac{2 \pi a p}{q}\right) i}+e^{\left(\frac{\pi s}{2}-\frac{2 \pi a p}{q}\right) i}\right) \zeta\left(s, \frac{a}{q}\right) \\
=i \frac{\Gamma(1-s)}{(2 \pi)^{1-s}}\left(e^{-\pi i s}-e^{\pi i s}\right) \zeta\left(1-s, \frac{p}{q}\right)
\end{gathered}
$$


or

$$
\begin{aligned}
2 q^{-s} & \sum_{a=1}^{q} \cos \left(\frac{\pi s}{2}-\frac{2 \pi a p}{q}\right) \zeta\left(s, \frac{a}{q}\right) \\
& =\frac{2 \Gamma(1-s) \sin \pi s}{(2 \pi)^{1-s}} \zeta\left(s, \frac{a}{q}\right)=\frac{2 \pi}{(2 \pi)^{1-s} \Gamma(s)} \zeta\left(s, \frac{a}{q}\right)
\end{aligned}
$$

whence (1.11) follows upon using the functional equation for the gamma function.

Now (1.12) follows from (1.11) in view of the formula

$$
-n \zeta(1-n, x)=B_{n}(x) .
$$

Remark 1. (i) We note that the above deduction of (1.11) amounts to applying the Hurwitz formula (cf. (1.7))

$$
\zeta(1-s, x)=\frac{\Gamma(s)}{(2 \pi)^{s}}\left(e^{-\frac{\pi i s}{2}} l_{s}(x)+e^{\frac{\pi i s}{2}} l_{s}(1-x)\right) .
$$

We also note that the reciprocal relation for the gamma function is equivalent to the Hurwitz formula, while (1.13) is its consequence (cf. Vista, Chapter 5]).

(ii) As is noted in the first proof of [SC, Theorem 6.3, p. 336], it is rather simple to deduce the corresponding results on Euler polynomials from (1.12) by means of the relation

$$
E_{n}(x)=\frac{2}{n+1}\left(B_{n+1}\left(\frac{x+1}{2}\right)-B_{n+1}\left(\frac{x}{2}\right)\right) .
$$

We shall therefore just state the result corresponding to (1.11),

$$
\begin{aligned}
2^{s-1} & \left(\zeta\left(1-s, \frac{p+q}{2 q}\right)-\zeta\left(1-s, \frac{p}{2 q}\right)\right) \\
& =-\frac{2 \Gamma(s)}{(2 \pi q)^{s}} \sum_{j=1}^{q} \cos \left(\frac{\pi s}{2}-2 \pi \frac{2 j-1}{q} a\right) \zeta\left(s, \frac{2 j-1}{2 q}\right),
\end{aligned}
$$

which depends on the relation

$$
2^{-s} \zeta\left(s, \frac{x+1}{2}\right)-q^{-s} \zeta\left(s, \frac{x}{2}\right)=\sum_{n=0}^{\infty} \frac{(-1)^{n}}{(n+x)^{s}}=L\left(\frac{1}{2}, s, x\right) .
$$

\section{LiMiting CASES}

We now turn to the limiting case $s \rightarrow n$ of (1.9) with $1 \leq n \in \mathbb{Z}$. We note that recently Murty-Saradha MS used the Laurent expansion (2.5) at $s=1$ of (1.9) to deduce the Gauss formula for the digamma function.

To state our main result, we introduce Milnor's gamma function $\gamma_{n}(x)$ (Mil]) through

$$
\gamma_{1+n}(x)=\frac{\partial}{\partial n} \zeta(-n, x)
$$


Theorem 2. For $q>1, n \in \mathbb{N}$ and $p \in \mathbb{Z}$, we have

$$
\begin{aligned}
& q^{-n} \sum_{a=1}^{q-1} e^{2 \pi i \frac{a}{q} p} \zeta\left(n, \frac{a}{q}\right) \\
& =\frac{i^{n+1}}{(2 \pi)^{1-n}(n-1) !}\left\{\pi i \frac{B_{n}\left(\frac{p}{q}\right)}{n}+\gamma_{n}\left(\frac{p}{q}\right)-(-1)^{n} \gamma_{n}\left(1-\frac{p}{q}\right)\right\} \\
& =\left\{\begin{array}{l}
\frac{(-1)^{\frac{n+2}{2}}}{(2 \pi)^{1-n}(n-1) !}\left\{\pi \frac{B_{n}\left(\frac{p}{q}\right)}{n}-i\left(\gamma_{n}\left(\frac{p}{q}\right)-\gamma_{n}\left(1-\frac{p}{q}\right)\right)\right\}, 2 \mid n \\
\frac{(-1)^{\frac{n+1}{2}}}{(2 \pi)^{1-n}(n-1) !}\left\{i \pi \frac{B_{n}\left(\frac{p}{q}\right)}{n}+\gamma_{n}\left(\frac{p}{q}\right)+\gamma_{n}\left(1-\frac{p}{q}\right)\right\}, 2 \not n,
\end{array}\right.
\end{aligned}
$$

the case $n=1$ being a singular case to be stated in Corollary 4 .

Corollary 3. For $2 \mid n$, the real and imaginary parts of (2.2) lead to Corollary 1 respectively, while for 2 \n, the imaginary and the the real parts respectively provide:

$$
\begin{aligned}
q^{-n} & \sum_{a=1}^{q}\left(\sin 2 \pi \frac{a}{q} p\right) \zeta\left(n, \frac{a}{q}\right) \\
& =\frac{(-1)^{\frac{n+2}{2}}}{(2 \pi)^{1-n}(n-1) !}\left(\gamma_{n}\left(1-\frac{p}{q}\right)-\gamma_{n}\left(\frac{p}{q}\right)\right), 2 \mid n, \\
q^{-n} & \sum_{a=1}^{q}\left(\cos 2 \pi \frac{a}{q} p\right) \zeta\left(n, \frac{a}{q}\right) \\
& =\frac{(-1)^{\frac{n+1}{2}}}{(2 \pi)^{1-n}(n-1) !}\left(\gamma_{n}\left(\frac{p}{q}\right)-\gamma_{n}\left(1-\frac{p}{q}\right)\right), 2 \not \chi n .
\end{aligned}
$$

Remark 2. The referee has kindly pointed out Lewin's paper [Le, pp. 355-375], in which one finds [Le, (16.30)] is derived directly from (1.6), and Corollary 3 coincides with [Le, (16.30), (16.31)].

Now by the Laurent expansion of $\zeta(s, x)$ at $s=1$,

$$
\zeta(s, x)=\frac{1}{s-1}-\psi(x)+O(s-1),
$$

where $\psi(x)$ designates the Euler digamma function

$$
\psi(x)=\frac{\Gamma^{\prime}}{\Gamma}(x),
$$

and Lerch's formula ([SC, (17), p. 92])

$$
\zeta^{\prime}(0, x)=\log \frac{\Gamma(x)}{\sqrt{2 \pi}}
$$

Theorem 2 in the case $n=1$ reads as (see the proof in $\S 3$ ):

Corollary 4 (Gauss' second formula ([SC, (49), p. 19])). We have

$$
\sum_{a=1}^{q} e^{2 \pi i \frac{a}{q} k} \psi\left(\frac{a}{q}\right)=q \log \left(1-e^{2 \pi i \frac{k}{q}}\right) .
$$




\section{Proof of Theorem 2}

Proof. We equate the limits of both sides of (1.9) as $s \rightarrow n$. First, we note that the left-hand side of (1.9) in the limit as $s \rightarrow n(n>1)$ becomes

$$
q^{-n} \sum_{a=1}^{q-1} e^{2 \pi i \frac{a}{q} p} \zeta\left(n, \frac{a}{q}\right)
$$

i.e. the left-hand side of (2.2). While if $n=1$, by the orthogonality

$$
\sum_{a=1}^{q} e^{2 \pi i \frac{a}{q}}=0
$$

the limit is

$$
-q^{-1} \sum_{a=1}^{q} e^{2 \pi i \frac{a}{q}} \psi\left(\frac{a}{q}\right)
$$

i.e. the left-hand side of (2.8). To take the limit of the right-hand side of (1.9), we recall

$$
\Gamma(1-s)=\frac{\frac{(-1)^{n}}{(n-1) !}}{s-n}+\frac{(-1)^{n-1}}{(n-1) !} \psi(n)+O(s-n), s \rightarrow n .
$$

Using (3.1) and

$$
\zeta(1-s, x)=-\frac{B_{n}(x)}{n}+\gamma_{n}(x)(s-n)+\cdots,
$$

we have

$$
\begin{aligned}
& \Gamma(1-s) e^{-\frac{\pi i s}{2}} \zeta(1-s, x)=\frac{1}{s-n} \frac{(-1)^{n}}{(n-1) !}(-i)^{n}\left(-\frac{B_{n}(x)}{n}\right)+(-i)^{n} \\
& \quad \times\left\{\frac{(-1)^{n-1}}{(n-1) !} \psi(n)\left(-\frac{B_{n}(x)}{n}\right)-\frac{\pi i}{2} \frac{(-1)^{n}}{(n-1) !}\left(-\frac{B_{n}(x)}{n}\right)+\frac{(-1)^{n}}{(n-1) !} \gamma_{n}(x)\right\}
\end{aligned}
$$

and correspondingly

$\Gamma(1-s) e^{\frac{\pi i s}{2}} \zeta(1-s, 1-x)=\frac{1}{s-n} \frac{(-1)^{n}}{(n-1) !} i^{n}\left(-\frac{B_{n}(x)}{n}\right)+i^{n}$

$$
\times\left\{\frac{(-1)^{n-1}}{(n-1) !} \psi(n)\left(-\frac{B_{n}(x)}{n}\right)+\frac{\pi i}{2} \frac{(-1)^{n}}{(n-1) !}\left(-\frac{B_{n}(1-x)}{n}\right)+\frac{(-1)^{n}}{(n-1) !} \gamma_{n}(x)\right\},
$$

whence

$$
\begin{aligned}
\lim _{s \rightarrow n} i \frac{1}{(2 \pi)^{1-s}} \Gamma(1-s)\left\{e^{-\frac{\pi i s}{2}} \zeta(1-s, x)-e^{\frac{\pi i s}{2}} \zeta(1-s, 1-x)\right\} \\
=\frac{i^{n+1}}{(2 \pi)^{1-n}(n-1) !}\left\{\pi i \frac{B_{n}(x)}{n}+\gamma_{n}(x)-(-1)^{n} \gamma_{n}(1-x)\right\}
\end{aligned}
$$

here $x=\frac{p}{q}$. Now (3.5) leads to the first equality of (2.2), and the second equality follows by distinguishing the parity of $m$. 
Proof of Corollary 4. The right-hand side of (3.5) gives (as $s \rightarrow 1$ )

$$
-\pi i B_{1}\left(\frac{p}{q}\right)+\zeta^{\prime}\left(0, \frac{p}{q}\right)+\zeta^{\prime}\left(0,1-\frac{p}{q}\right) .
$$

Using (2.7) and the reciprocal relation, we get $(0<x<1)$

Writing

$$
\begin{aligned}
\zeta^{\prime}(0, x)+\zeta^{\prime}(0,1-x) & =\log \frac{\Gamma(x) \Gamma(1-x)}{2 \pi} \\
& =\log \frac{1}{2 \pi \sin \pi x} .
\end{aligned}
$$

we see that

$$
\zeta^{\prime}(0, x)+\zeta^{\prime}(0,1-x)=\pi i\left(x-\frac{1}{2}\right)-\log \left(1-e^{2 \pi i x}\right)=\pi i \overline{B_{1}}(x) .
$$

Hence, the limit of the right-hand side of (1.8) is

$$
-\log \left(1-e^{2 \pi i \frac{p}{q}}\right) \text {. }
$$

Comparing these limit values, we conclude (2.8).

Remark 3. Integrating [Mil, (25)], we obtain

$$
\gamma_{2}(x)-\gamma_{2}(1-x)=-\int_{1}^{x} \log 2 \sin \pi t d t+C .
$$

Similarly, as in [Mil, p. 313], each $\gamma_{n}$ is essentially an indefinite integral of $\gamma_{n-1}$ up to a constant factor and a Bernoulli polynomial summand, and so the odd part $\gamma_{n}(x)-\gamma_{n}(1-x)$ is essentially the Clausen function mentioned in $\S 1$ (cf. [K] p. 469]).

Thus, we see that Theorem 2 captures the behavior of the Clausen functions, which escapes the seizure of Corollary 1. Indeed, $\gamma_{n}(x)$ are iterated integrals of $\log \Gamma(x)$ and therefore are closely connected to Barnes' multiple gamma functions.

\section{ACKNOWLEDGMENT}

We are grateful to the referee for enlightening comments which enabled us to make the paper more orderly.

\section{REFERENCES}

[Ap] T. M. Apostol, Introduction to analytic number theory, Springer-Verlag, New YorkHeidelberg, 1976. MR0434929 (55:7892)

[CK1] D. Cvijović and J. Klinowski, New formula for the Bernoulli and Euler polynomials at rational arguments, Proc. Amer. Math. Soc. 123 (1995), 1527-1535. MR1283544(95g:11085)

[CK2] D. Cvijović and J. Klinowski, Closed form summation of some trigonometric series, Math. Comput. 64 (1995), 205-210. MR.1270616 (95f:65017)

[K] S. Kanemitsu, On evaluation of certain limits in closed form, Théorie des nombres, ed. by J.-M. De Koninck and C. Levesque, Walter de Gruyter, Berlin-New York, 1989, 459-474. MR 1024583 (90m:11127)

[Vista] S. Kanemitsu and H. Tsukada, Vistas of special functions, World Scientific, Hackensack, NJ, 2007. MR 2352572

[Le] L. Lewin, Polylogarithms and associated functions, North-Holland, New York, 1981. MR618278 (83b:33019) 
[LHK] H.-L. Li, M. Hashimoto and S. Kanemitsu, On structural elucidation of Eisenstein's formula, to appear.

[Mil] J. Milnor, On polylogarithms, Hurwitz zeta-functions and the Kubert identities, Enseign. Math. (2) 29 (1983), 281-322. MR719313 (86d:11007)

[MS] M. Ram Murty and N. Saradha, Transcendental values of the digamma function, J. Number Theory 125 (2007), 298-318. MR2332591 (2008g:11123)

[S1] H. M. Srivastava, A note on the closed-form summation of some trigonometric series, Kobe J. Math. 16 (1999), 177-182. MR1745023 (2000m:11080)

[S2] H. M. Srivastava, Some formulas for the Bernoulli and Euler polynomials at rational arguments, Math. Proc. Camb. Philos. Soc. 129 (2000), 77-84. MR.1757780(2001f:11033)

[SC] H. M. Srivastava and J. Choi, Series associated with the zeta and related functions, Kluwer Academic Publishers, Dordrecht-Boston-London, 2001. MR1849375 (2003a:11107)

[Y] Y. Yamamoto, Dirichlet series with periodic coefficients, Proc. Intern. Sympos. "Algebraic Number Theory", Kyoto, 1976, Japan Soc. Promotional Sci., Tokyo, 1977, 275-289. MR0450213(56:8509)

Harish-Chandra Research Institute, Chhatnag Road, Jhusi, Allahabad 211 019, India

E-mail address: kalyan@hri.res.in

Graduate School of Advanced Technology, Kinki University, Iizuka, Fukuoka 8208555, JAPAN

E-mail address: kanemitu@fuk.kindai.ac.jp

Department of Mathematics, Weinan Teachers College, Weinan, Shaanxi, 714000, People's Republic of China

E-mail address: lihailong@wntc.edu.cn 